\title{
Assessing Knowledge Management in the Power Sector through a Connectionist Model
}

\author{
Álvaro Herrero, Lourdes Sáiz, and Emilio Corchado
}

\begin{abstract}
It has been proven that Artificial Intelligence, in general, and Artificial Neural Networks, in particular, can be successfully applied to problems in the field of Knowledge Management (KM). One such problem is the identification and assessment of a company's KM status. Nowadays the importance of KM to organisational survival and for the maintenance of competitive strength is widely acknowledged. Several connectionist models for the assessment and analysis of KM status are proposed and applied in this work. These models account for the specific features of a company in the Energy sector/Power sector: a dynamic, essential service and one of the basic pillars that supports the so-called "welfare state", constituting an established strategic sector in any globalized economy.
\end{abstract}

Keywords: Knowledge Management, Exploratory Projection Pursuit, Maximum Likelihood Hebbian Learning, Energy/Power Sector.

\section{Introduction}

This study of Knowledge Management (KM) centres its attention on the design, organisation and application of innovative and creative ways of resource management, and in particular, those of a more intangible nature [1], [2] until recently left out of traditional business management models. It contributes value to the firm, insofar as that refers to "knowing how to use" resources, rather than merely possessing them, which can achieve satisfactory management of the stock of knowledge that is already held or that may be developed by a firm.

Álvaro Herrero · Lourdes Sáiz

Civil Engineering Department, University of Burgos

C/ Francisco de Vitoria s/n, 09006 Burgos, Spain

e-mail: $\{$ ahcosio, 1saiz $\}$ aubu.es

Emilio Corchado

2Departamento de Informática y Automática, University of Salamanca,

Plaza de la Merced s/n, 37008 Salamanca, Spain

e-mail: escorchado@ubu.es

Y. Demazeau et al. (Eds.): Trends in PAAMS, AISC 71, pp. 721-729.

springerlink.com

(c) Springer-Verlag Berlin Heidelberg 2010 
The application of KM involves the deployment of a set of procedures that allow a firm, on a case-by-case basis, to acquire, capitalize, retain, transfer, and use its strategic knowledge [3]. In essence, the purpose of KM is to identify, acquire, transform, develop, disseminate, use and preserve knowledge that is relevant to achieve specific short and long-term objectives, preferably for the development of new opportunities [4].

On this point, we should set out the differences in terms of "datum", "information " and "knowledge". Data are located in the world, and knowledge is held by the agents (people/organisations). Information is the nexus between both. Knowledge that is acquired, broadened, reviewed, contrasted and used by people in the firm, throughout their working life, is of the greatest value to the organization. Knowledge not only represents the cause, but the result, the outcome and the success of the business activity. In addition, its wealth lies in the variety of knowledge that the firm can amass, apply and develop in its day-to-day activities, along with the social or collective nature of the knowledge, given that a firm's knowledge is not merely a juxtaposition of the individual knowledge of its employees, but a construct that emerges from the events experienced by the firm and its working methods, organizational routines and common values, developed over time.

The identification of knowledge in the form of patterns that exist across dimensional boundaries in high dimensional datasets is a challenging task. Such patterns may become visible if changes are made to the spatial coordinates. Projection models perform such changes by projecting high-dimensional data onto a lower dimensional space in order to identify "interesting" directions in terms of a specific index or projection. These indexes or projections are, for example, based on the identification of directions that account for the largest variance of a dataset which is the case of Principal Component Analysis (PCA) [5], [6] or the higher order statistics such as the skew or kurtosis index -which is the case of Exploratory Projection Pursuit (EPP) [7]. Having identified the most interesting projections, the data is then projected onto two or three dimensions, which allows its structure to be examined with the naked eye. In this study, several projection models are applied as a KM tool to the Energy/Power Market.

The rest of this paper is organized as follows. Section 2 overviews KM in the Energy/Power sector, while Section 3 describes the main connectionist model applied in this work. Section 4 presents the experimental domain and the results from different unsupervised models. Finally, section 5 arrives at general conclusions and describes future work.

\section{Knowledge Management in the Energy/Power Market}

The energy sector in the Spanish economy has quite specific features that more than justify the design and application of management models based on the knowledge of the people that constitute the firms in this industry.

Among its special characteristics, the sector's close relationship with a country's industrialization process may be highlighted, its strategic value, with strong "trickle-down effects" for other industries and economic activities, and its tendency to behave according to non-competitive market models. Moreover, energy 
is implicit in one of the basic pillars that supports the so-called "welfare state", constituting an established strategic sector in any globalized economy.

Owing to the high degree of energy dependence observed in Spain and in Spanish firms, and the broad typology of problems that arise, the efficient application of KM systems is of even greater interest, in order to achieve the correct generation of knowledge, and its transmission, use and adaption to change, which will ensure a satisfactory and reliable supply of electricity. At the same time, the energy sector holds associated knowledge, values that underpin its organizational culture, part of its know-how and technical and organizational capacity, which are unique and are not found in other sectors. Along with this, a great part of the knowledge and ability needed to develop the work in an effective and, above all, in a safe way can only be acquired within the sector. A great amount of this knowledge and these abilities need years of training and experience.

The introduction of a KM model in energy firms [8], [9], will enable key, sector-specific knowledge and abilities to be identified. It will facilitate their correct generation and complete application, the inventory of expert people, the retention and capitalization of experience and "know how", beyond that held by the expert, sharing and transfer between the people involved and the need for training and updating, arising from new needs that appear every day, such as regulatory requirements, improvements in technical capacities or greater operative experience.

\section{Connectionist Projection Model}

The solution proposed in this paper applies an unsupervised neural model called Cooperative Maximum Likelihood Hebbian Learning (CMLHL) [10], [11]. It is based on Maximum Likelihood Hebbian Learning (MLHL) [10], [12]. Considering an N-dimensional input vector $(x)$, and an M-dimensional output vector $(y)$, with $W_{i j}$ being the weight (linking input $j$ to output $i$ ), then CMLHL can be expressed as:

1. Feed-forward step: $y_{i}=\sum_{j=1}^{\mathrm{N}} W_{i j} x_{j}, \forall i$

2. Lateral activation passing: $y_{i}(t+1)=\left[y_{i}(t)+\tau(b-A y)\right]^{+}$

3. Feedback step: $e_{j}=x_{j}-\sum_{i=1}^{M} W_{i j} y_{i}, \forall j$

4. Weight change: $\Delta W_{i j}=\eta \cdot y_{i} \cdot \operatorname{sign}\left(e_{j}\right)\left|e_{j}\right|^{p-1}$

Where: $\eta$ is the learning rate, $\tau$ is the "strength" of the lateral connections, $b$ the bias parameter, $p$ a parameter related to the energy function [10], [11], [12] and $A$ a symmetric matrix used to modify the response to the data [11]. The effect of this matrix is based on the relation between the distances separating the output neurons. 


\section{Case Study}

The empirical study presented in this work is based on the analysis of a set of data related to the introduction of KM in an energy sector firm, using projection methods. The data sample totals 229 representative records (samples) on the status of critical knowledge and its management in the firm under study.

Each record covers information on the task or function assigned to each employee and the knowledge that is needed to carry out that task correctly. The tasks are analyzed according to their degree of difficulty, their impact on the business, who receives their results and the way in which they are received. In contrast, the characteristics of the knowledge under study are difficulty of transfer, its importance to the work, whether it is documented, the knowledge level of the expert, the degree to which it is known and shared with other employees, the method used to transfer it and the urgency of its acquisition, in case of a shortfall or a lower level than that required being reached. It should be pointed out that because of the type of firm under study, the parameters on the degree of documentation and the knowledge level held were not discriminatory, which is to say, the necessary key knowledge is held within the firm and is documented.

In this study, the objective of the analysis is to cross-reference the data against common characteristics or parameters, from both the tasks and their associated knowledge, in order to construct a representative map of tasks and the key knowledge and their immediate visualization, according to their situation on the map. All of that will serve to take more accurate decisions when setting up a KM system in harmony with the firm's selected strategy.

\subsection{Description of the Problem under Study}

The data sample under study amounts to 229 records that correspond to a total of 120 tasks/functions and 142 critical pieces of knowledge gathered from among the employees of a firm. This dataset represents the knowledge state of the firm and, once the data is processed and the corresponding conclusions drawn, these lead to proposals for actions that need to be taken in relation to retention, protection and capitalization of key knowledge for the competitiveness of the business.

For each of the tasks, the knowledge state comprises the aforementioned information referring to the degree of difficulty associated with its execution, the set of knowledge that is applied, its impact on key aspects of the firm, who will receive the result of carrying out each task and how it will be received. This set of data is completed with the detail and the characteristics presented by the knowledge that is assigned to each task and the data relating to the receptor of the knowledge. Thus, the following features are gathered for each piece of knowledge:

- Level of knowledge acquired, ranging from 1 (not enough) to 4 (expert).

- Difficulty of the knowledge, ranging from 1 (lowest) to 5 (highest).

- Receptors of the knowledge, generating 4 binary features (section/department/company/external partners). 
- Documented or otherwise, binary values: 1 (documented) and 0 (undocumented).

- Importance to the task, ranging from 1 (medium) to 5 (critical).

- Urgency of knowledge transfer, ranging from 1 (6 months) to 5 (immediate).

- Difficulty of knowledge transfer, ranging from 1 (low) to 5 (high).

- Method of transfer, generating 6 binary features (Written/Oral/On-the-job observation/Training/Inactive/Practicing).

- Number of people that share the knowledge.

- Receptor required training level, ranging from 0 (none) to 2 (university degree).

- Consequence of knowledge absence, ranging from 1 (lowest) to 5 (highest).

As a result, 24 features for each one of the records are included in the dataset.

\subsection{Experiments and Results}

CMLHL was applied in order to analyse the dataset described above and to identify its inner structure. The best CMLHL projection is shown in Fig. 1.

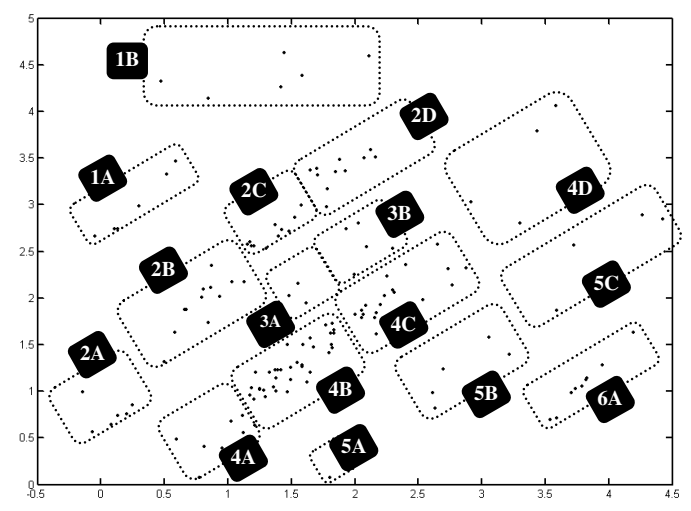

Fig. 1 CMLHL projection

The following interesting conclusions may be drawn from the position of the points (knowledge-tasks) on the projection. Starting with the "Importance of knowledge to the task", of the three possible options -indispensable, important and average- it may be seen that the points that are found in the upper third of the projection come under the heading of "indispensable", the central positions represent important knowledge and those in the lower third are of average importance. The results show that the largest part of the identified knowledge is either important or is of average importance.

With regard to "Knowledge sharing" within the firm, it shows records that go from less to more; less dispersed knowledge is situated in the upper part of the projection and the most widely shared knowledge, in the lower part. Linking this 
latter result with the former one, it may be concluded that the least important knowledge is the most widely shared, the contrary being true in the case of indispensable knowledge.

The variable "Difficulty of knowledge transfer" covers three possible values that are high, medium and low. High difficulty is represented by the points in the upper third of the projection, the central area covers knowledge of medium difficulty and the points in the lower third represent knowledge of the lowest difficulty. It may therefore be seen that most of the knowledge under analysis has a transfer difficulty of between medium and low, as well as presenting intermediate levels of importance and a high degree of sharing.

The "Consequence of not holding the knowledge" provides information on the effects and their impact on the tasks due to a lack of necessary knowledge; on a barometer of serious, moderate and minor. Here, the distribution of the points reveals that the knowledge situated to the mid-left of the projection presents serious consequences, a parameter which changes as we move to the right.

With regard to the characteristic that refers to "Urgency for knowledge transfer", three possible responses were foreseen that are 'immediate', 'six-months' and 'over six-months'. CMLHL allows us to observe a vertical trend by which the upper positions reflect an immediate urgency, which moving down to the centre drops to 6 months, and continues to drop down to lower levels, reaching the lowest value, which is over 6 months. Examining the conclusions reached up until this point, this last point on the urgency of transfer shows that critical knowledge is the least shared, presents the greatest difficulty in its transfer and, in addition, is revealed to be of immediate urgency. For its part, knowledge of average importance neither presents as much difficulty, nor is such a short time required for its transfer and, furthermore, the analysis revealed that it is more evenly shared than the other types of knowledge.

With regard to the "Method of knowledge transfer", the positions of the points allow us to conclude very clearly that those on the left represent situations centred on "training" and "on-the-job observation"; knowledge that is more efficiently shared through "practicing" is positioned in the centre and the "written" and "oral" methods are positioned to the right.

Integrating the earlier results on the areas in which the knowledge may be grouped (1A, 1B, 2A, 2B, 2C, 2D, 3A, 3B, 4A, 4B, 4C, 4D, 5A, 5B, 6A), according to its position (Fig. 1), the earlier analysis may be complemented by looking at the features and consequences presented by the knowledge according to the position in each of the aforementioned sets.

The knowledge in group 1A is indispensable, there is a slight tendency to sharing, whereas the consequences of its absence are high, the difficulty of transfer is equally high and the urgency of the knowledge that is not held is almost immediate. This set of data represents a weak situation with regard to sharing and diffusion of knowledge within the firm.

Something very similar may be said about the points that make up group $1 \mathrm{~B}$, except that its difficulty of transfer is not so clearly appreciated and sharing is still less than in group 1A. Equally, such results represent situations in which 
knowledge is lacking, and they hold negative consequences for the firm in the case of a lack of knowledge.

Knowledge of average importance is found around the fringes of $2 \mathrm{~A}$, which is reasonably well shared, its difficulty of transfer is minor, as is the case for the urgency of its transfer and the consequences of a lack of knowledge are situated between serious and moderate. All the parameters, except the importance of knowledge, reveal a situation that is close to optimal. As shown in the comparison between groups $1 \mathrm{~A}$ and $1 \mathrm{~B}$, in this case, the situations that $2 \mathrm{~B}$ and $2 \mathrm{C}$ represent are almost identical to those described in $2 \mathrm{~A}$; in both the exception is given by the degree of importance attributed to knowledge that is a little greater, and the same may be said for the difficulty of its transfer.

Group 2D brings together the strategic knowledge for the tasks, which is not however shared between the employees; its difficulty being high and its urgency of transfer serious. This group reveals the need to adopt the necessary means to capitalize and share key knowledge, either through training, practicing with the expert, overlapping activities with the others or temporarily changing activities.

Groups $3 \mathrm{~A}$ and $3 \mathrm{~B}$ coincide in that they both have indispensable and important knowledge, although these are more evenly shared in $3 \mathrm{~A}$, and likewise the difficulty is lower and its urgency of transfer is minor. The consequences of a lack of knowledge are presented at almost the same level, which is medium.

Less importance is given to knowledge in Group 4A: it is shared, the difficulty of transfer is low and the urgency for the knowledge is not immediate. Thus, the study reveals that the consequences of a lack of knowledge, where applicable, reach an intermediate value. The records found in groups $4 \mathrm{~B}$ and $4 \mathrm{C}$, both represent very similar situations: $4 \mathrm{C}$ has a slightly better situation with respect to the importance of knowledge for the task, but a worse one with respect to lower values for sharing knowledge and better results under the heading of difficulty of transfer. The group 4D, has notably better results for the quality of knowledge, but worse results for its sharing and difficulty of transfer with respect to $4 \mathrm{C}$.

Group 5A contains a single point that can be considered an outlier, while group 5B brings together knowledge of medium importance for the tasks that is fully shared, and that has a transfer difficulty of almost zero and the lowest possible urgency. The consequences of its absence are very minor. It is verging on an optimal situation. In contrast, knowledge is more important in 5C, although it is also shared and the difficulty of its transfer is a little greater.

Finally, group 6A brings together the most important but not indispensable knowledge, which is largely shared within the firm, whereas its difficulty and urgency of transfer reach lower values and the consequences of its absence are minor.

\subsection{Comparative Study}

For comparative purposes, some other projection techniques, namely PCA (see section 4.3.1) and MLHL (see section 4.3.2), were applied to the previously described dataset. The obtained projections are shown in Fig. 2. 


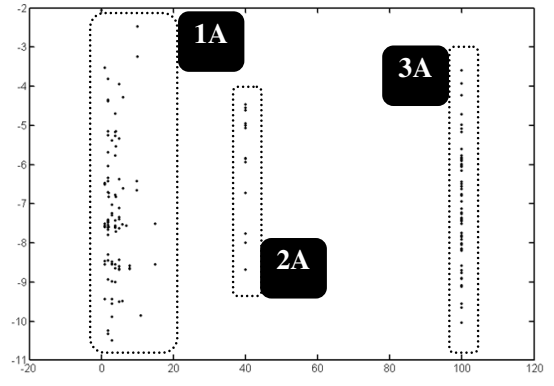

a) PCA projection.

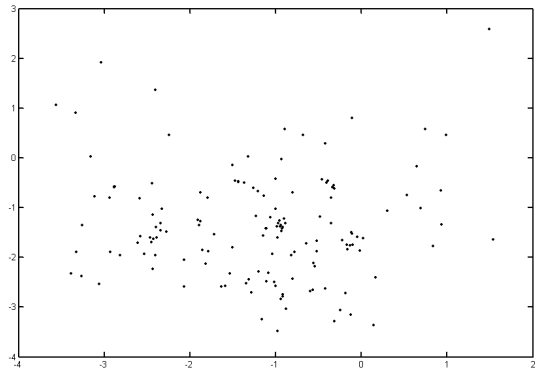

a) MLHL projection.

Fig. 2 Projections of the analysed dataset

As can be seen in Fig. 2.a, the PCA [5] [6] projection is able to identify a general structure of the dataset consisting of 3 main groups (1A, 2A, and 3A). After analysing the samples in each one of these groups, it can be concluded that only the feature "Number of people that share the knowledge" in the original dataset was taken into account when projecting that structure. This feature provides the greatest variance in the dataset and, as a result, PCA has identified it as the first principal component.

As can be seen in Fig. 2.b, the MLHL projection is not as good as the CMLHL projection (Fig. 1), as it shows the inner structure of the dataset less clearly. MLHL provides a sparse projection of the data but sharply defined groups can not be identified as in the case of CMLHL.

\section{Conclusions and Future Work}

An assessment of KM is especially critical in the Energy/Power sector. This work has proposed a tool based on a connectionist model for the assessment and analysis of the KM status in a company which takes the specific features of the Energy/Power sector into account. As can be seen in section 4.2, the CMLHL model enabled KM managers to describe the KM status of a power company. This can be used to analyse and consequently improve the KM process of the whole company. It has been also shown that CMLHL outperforms PCA and MLHL in obtaining the most useful projection of a given dataset for KM purposes.

Future work will focus on the upgrade of the proposed model to automate the proposals to improve the KM status by applying different techniques in a Hybrid Artificial Intelligence System.

Acknowledgments. This research is partially supported through the Junta de Castilla and León project BU006A08, Business intelligence for production within the framework of the Instituto Tecnológico de Castilla y León (ITCL) and the Agencia de Desarrollo Empresarial (ADE), and the Spanish Ministry of Education and Innovation project CIT020000-2008-2. The authors would also like to thank the vehicle interior manufacturer, 
Grupo Antolin Ingenieria S.A., within the framework of the project MAGNO2008 - 1028.CENIT Project funded by the Spanish Government.

\section{References}

1. Collis, D.J., Montgomery, C.A.: Competing on Resources: Strategy in the 1990s. Harv. Bus. Rev. 73(4), 118-128 (1995)

2. Maranto-Vargas, D., Rangel, R.G.T.: Development of Internal Resources and Capabilities as Sources of Differentiation of SME under Increased Global Competition: A Field Study in Mexico. Technol. Forecast. Soc. Chang. 74(1), 90-99 (2007)

3. Wiig, K.M.: Knowledge Management Foundations: Thinking about Thinking-how People and Organizations Represent, Create, and Use Knowledge. Schema Press, Limited (1994)

4. Soo, C.W., Devinney, T.M., Midgley, D.F.: External Knowledge Acquisition, Creativity and Learning in Organisational Problem Solving. International Journal of Technology Management 38(1), 137-159 (2007)

5. Pearson, K.: On Lines and Planes of Closest Fit to Systems of Points in Space. Philosophical Magazine 2(6), 559-572 (1901)

6. Oja, E.: Neural Networks, Principal Components, and Subspaces. International Journal of Neural Systems 1, 61-68 (1989)

7. Friedman, J.H., Tukey, J.W.: A Projection Pursuit Algorithm for Exploratory DataAnalysis. IEEE Transactions on Computers 23(9), 881-890 (1974)

8. Metaxiotis, K.: Better Management of Energy Knowledge: the Key for Success in the Energy Sector. International Journal of Computer Applications in Technology 22(2/3), 104-108 (2005)

9. Carpenter, S., Rudge, S.: Benchmarking KM at British Energy. Knowledge Management 6(6), 3 (2003)

10. Corchado, E., MacDonald, D., Fyfe, C.: Maximum and Minimum Likelihood Hebbian Learning for Exploratory Projection Pursuit. Data Mining and Knowledge Discovery 8(3), 203-225 (2004)

11. Corchado, E., Fyfe, C.: Connectionist Techniques for the Identification and Suppression of Interfering Underlying Factors. International Journal of Pattern Recognition and Artificial Intelligence 17(8), 1447-1466 (2003)

12. Fyfe, C., Corchado, E.: Maximum Likelihood Hebbian Rules. In: 10th European Symposium on Artificial Neural Networks, ESANN 2002 (2002) 
